\title{
A new variant of symmetry breaking for quark mass matrices
}

\section{Citation}

Lehmann, Harry, Conrad Newton, and Tai Tsun Wu. 1996. "A New Variant of Symmetry

Breaking for Quark Mass Matrices." Physics Letters B 384 (1-4): 249-54. https://

doi.org/10.1016/0370-2693(96)00609-0.

\section{Permanent link}

http://nrs.harvard.edu/urn-3:HUL.InstRepos:41555803

\section{Terms of Use}

This article was downloaded from Harvard University's DASH repository, and is made available under the terms and conditions applicable to Other Posted Material, as set forth at http:// nrs.harvard.edu/urn-3:HUL.InstRepos:dash.current.terms-of-use\#LAA

\section{Share Your Story}

The Harvard community has made this article openly available.

Please share how this access benefits you. Submit a story.

Accessibility 


\title{
A New Variant of Symmetry Breaking for Quark Mass Matrices
}

\author{
Harry Lehmann \\ II. Institut für Theoretische Physik der Universität Hamburg, Hamburg, Germany \\ Conrad Newton* and Tai Tsun $\mathrm{Wu}^{*}$ \\ Gordon McKay Laboratory, Harvard University, Cambridge, Massachusetts 02138, USA \\ and \\ Theoretical Physics Division, CERN, CH 1211 Geneva 23, Switzerland
}

\begin{abstract}
We propose a definite pattern for the breaking of a discrete flavor symmetry of the quark mass matrices which is caused by the masses of the first two generations of quarks. We discuss the consequences of this proposal for the KM matrix.
\end{abstract}

*Work supported in part by the United States Department of Energy under Grant No. DE-FG02-84ER40158.

CERN-TH/95-240

September 1995 
1. In this note, we try to express some parameters of the KM matrix $V$ [1] in terms of the other parameters of the standard model. This approach is guided by the experimental information on $\mathrm{V}$ and a desire to have a simple pattern of flavor symmetry breaking. In a current basis, the charged hadronic currents are

$$
J_{\mu} \sim \bar{u}_{L} \gamma_{\mu} d_{L}
$$

where

$$
d=\left(\begin{array}{l}
d_{1} \\
d_{2} \\
d_{3}
\end{array}\right), \quad u=\left(\begin{array}{l}
u_{1} \\
u_{2} \\
u_{3}
\end{array}\right)
$$

denote the $d$ and $u$ quark fields. We use $q$ for quark fields when no distinction between $d$ and $u$ quarks is necessary. The quark mass matrices $\mathrm{M}(d)$ and $\mathrm{M}(u)$, obtained by taking the vacuum expectation value of the Higgs field in the quark-Higgs coupling, are

$$
\bar{d}_{L} \mathrm{M}(d) d_{R}+\bar{u}_{L} \mathrm{M}(u) u_{R}+\text { h.c. }
$$

We take $\mathrm{M}(d)$ and $\mathrm{M}(u)$ as hermitean matrices. Application of the same unitary transformation to $\mathrm{M}(d)$ and $\mathrm{M}(u)$ leaves (1) invariant.

2. Following Fritzsch [2], we make the following assumption.

Assumption 1:

a) If $M(d)$ and $M(u)$ both have one zero eigenvalue, then there exists a current basis for the quark fields such that

$$
\begin{array}{lllll}
\mathrm{M}_{i j}(d)=0 & \text { for } & i=1 & \text { or } & j=1 \\
\mathrm{M}_{i j}(u)=0 & \text { for } & i=1 & \text { or } & j=1
\end{array}
$$

b) If $\mathrm{M}(d)$ and $\mathrm{M}(u)$ both have two zero eigenvalues, there exists a current basis for the quark fields such that the only non-zero elements of $\mathrm{M}(d)$ and $\mathrm{M}(u)$ are $\mathrm{M}_{33}(d)$ and $\mathrm{M}_{33}(u)$.

We call this a heavy basis. Moreover, we assume that the elements of $M(d)$ and $M(u)$ are continuous functions of the quark masses.

An important consequence of Assumption 1 is the existence of a partial flavor (or horizontal) symmetry. This is a permutation symmetry which is broken by the masses of the second and first generations. As has been noted by several authors [3], the symmetry is easily visible if we pass from a heavy basis to a coherent basis which we shall do presently.

For the case that $\mathrm{M}(d)$ and $\mathrm{M}(u)$ have at least one zero eigenvalue each it follows from Assumption 1(a) that

$$
\mathrm{M}=\left(\begin{array}{ccc}
0 & 0 & 0 \\
0 & c & b \\
0 & b^{*} & a
\end{array}\right)
$$

for both $M(d)$ and $M(u)$. By continuity this $M$ can be written as a sum

$$
\mathrm{M}=\mathrm{M}_{3}+\mathrm{M}_{2}
$$


with

$$
\mathrm{M}_{3}=\left(m_{2}+m_{3}\right)\left(\begin{array}{lll}
0 & 0 & 0 \\
0 & 0 & 0 \\
0 & 0 & 1
\end{array}\right)
$$

and

$$
\mathrm{M}_{2}=\left(\begin{array}{ccc}
0 & 0 & 0 \\
0 & c & b \\
0 & b^{*} & -c
\end{array}\right)
$$

where $\mathrm{M}_{2}$ approaches zero as $m_{2}(d) \rightarrow 0$ and $m_{2}(u) \rightarrow 0$.

3. It is instructive to review previous work [4] which was done for the first two generations only. This can be viewed as the limit $m_{3} \rightarrow \infty$ of the general case. For two generations we have $2 \times 2$ matrices $\mathrm{M}^{(2)}(d)$ and $\mathrm{M}^{(2)}(u)$, which are in a heavy basis

$$
\mathrm{M}^{(2)}=\left(\begin{array}{cc}
c & b \\
b^{*} & a
\end{array}\right)
$$

Fritzsch [4] made the assumption that there exists a heavy basis where

$$
c(d)=c(u)=0
$$

With

$$
\mathrm{M}_{\text {diag }}^{(2)}=\operatorname{diag}\left(m_{1},-m_{2}\right),
$$

it follows from this assumption that

$$
a=-m_{2}+m_{1} \quad \text { and } \quad|b|^{2}=m_{1} m_{2} .
$$

This determines the Cabibbo angle [5] given by

$$
\sin \theta_{c}=\left|V_{12}^{(2)}\right|
$$

in terms of $\frac{m_{d}}{m_{s}}, \frac{m_{u}}{m_{c}}$ and a phase

$$
\phi=|\phi(d)-\phi(u)|
$$

where $\phi(d)=\arg b(d)-\pi, \phi(u)=\arg b(u)-\pi$. To obtain agreement with the experimental value of $\sin \theta_{c}$, the phase $\phi$ has to be chosen as

$$
\phi \simeq \frac{\pi}{2}
$$

For $\phi=\pi / 2$,

$$
\left|V_{12}^{(2)}\right|=\sqrt{\frac{\left(\frac{m_{d}}{m_{s}}+\frac{m_{u}}{m_{c}}\right)}{\left(1+\frac{m_{d}}{m_{s}}\right)\left(1+\frac{m_{u}}{m_{c}}\right)}}
$$


For our purpose it is of interest to transform

$$
\mathrm{M}^{(2)}=(a+c)\left(\begin{array}{ll}
0 & 0 \\
0 & 1
\end{array}\right)+\left(\begin{array}{cc}
c & b \\
b^{*} & -c
\end{array}\right)
$$

to a coherent basis. For two generations this amounts to defining

$$
\mathrm{M}_{c}^{(2)}=\mathrm{U}_{c}^{(2)}{ }^{\dagger} \mathrm{M}^{(2)} \mathrm{U}_{c}^{(2)}
$$

where

$$
\mathrm{U}_{c}^{(2)}=\left(\begin{array}{cc}
\frac{1}{\sqrt{2}} & -\frac{1}{\sqrt{2}} \\
\frac{1}{\sqrt{2}} & \frac{1}{\sqrt{2}}
\end{array}\right)
$$

$\mathrm{M}_{c}^{(2)}$ is given by

$$
\mathrm{M}_{c}^{(2)}=\frac{(a+c)}{2}\left(\begin{array}{ll}
1 & 1 \\
1 & 1
\end{array}\right)+\frac{1}{2}\left(\begin{array}{cc}
b+b^{*} & -2 c+b-b^{*} \\
-2 c-\left(b-b^{*}\right) & -\left(b+b^{*}\right)
\end{array}\right) .
$$

The first term of $\mathrm{M}_{c}^{(2)}$ has an $\mathrm{S}_{L}(2) \times \mathrm{S}_{R}(2)$ symmetry. This symmetry is broken by the second term, i.e. it is broken by the non-zero mass of the first generation. We observe that for the case $c(d)=c(u)=0$ the symmetry breaking term changes sign under the transformation

$$
q_{1 c} \leftrightarrow q_{2 c}
$$

Equivalently we can say that the symmetry is broken by a term which transforms as the antisymmetric representation of the diagonal subgroup of $\mathrm{S}_{L}(2) \times \mathrm{S}_{R}(2)$. We take this as a guide for the symmetry breaking in the case of three generations.

4. In this section we study the case where for three generations $M(d)$ and $M(u)$ have one zero eigenvalue each. We begin with a simple mathematical observation on a pair of $2 \times 2$ hermitean matrices, say $H_{1}$ and $H_{2}$. In such a case there exists a unitary transformation $U_{0}$ such that $U_{0}^{\dagger} H_{1} U_{0}$ and $U_{0}^{\dagger} H_{2} U_{0}$ are both real. The reason is that $H_{1}$ and $H_{2}$ can be expressed in terms of Pauli matrices

$$
\begin{aligned}
& H_{1}=s_{1}+\vec{v}_{1} \cdot \vec{\sigma} \\
& H_{2}=s_{2}+\vec{v}_{2} \cdot \vec{\sigma}
\end{aligned}
$$

Use $U_{0}$ to rotate the real vectors $\vec{v}_{1}$ and $\vec{v}_{2}$ such that they both lie in the 1-3 plane. This proves the above observation since the Pauli matrices $\sigma_{1}$ and $\sigma_{3}$ are real.

In this special heavy basis the matrices $\mathrm{M}_{2}(d)$ and $\mathrm{M}_{2}(u)$ of equation $(6)$ are

$$
\mathrm{M}_{2}=\left(\begin{array}{ccc}
0 & 0 & 0 \\
0 & c & b \\
0 & b & -c
\end{array}\right)
$$

with $b, c$ real. We transform $\mathrm{M}_{3}$ and $\mathrm{M}_{2}$ to a coherent basis by defining

$$
\mathrm{M}_{c}=\mathrm{U}_{c}^{\dagger} \mathrm{MU}_{c}
$$


where, analogous to $(10)$,

$$
\mathrm{U}_{c}=\left(\begin{array}{ccc}
\frac{1}{\sqrt{2}} & -\frac{1}{\sqrt{2}} & 0 \\
\frac{1}{\sqrt{6}} & \frac{1}{\sqrt{6}} & -\frac{2}{\sqrt{6}} \\
\frac{1}{\sqrt{3}} & \frac{1}{\sqrt{3}} & \frac{1}{\sqrt{3}}
\end{array}\right) .
$$

This transforms $\mathrm{M}_{3}$ and $\mathrm{M}_{2}$ into

$$
\mathrm{M}_{3 c}=\frac{\left(m_{2}+m_{3}\right)}{3}\left(\begin{array}{lll}
1 & 1 & 1 \\
1 & 1 & 1 \\
1 & 1 & 1
\end{array}\right)
$$

and

$$
\mathrm{M}_{2 c}=\frac{1}{3}\left(\begin{array}{ccc}
A & A & B \\
A & A & B \\
B & B & -2 A
\end{array}\right)
$$

with

$$
\begin{aligned}
& A=-\frac{c}{2}+\sqrt{2} b \\
& B=-2 c-\frac{b}{\sqrt{2}} .
\end{aligned}
$$

These formulae show that the mass matrices have a partial flavor symmetry: $M_{c}$ has an $\mathrm{S}_{L}(3) \times \mathrm{S}_{R}(3)$ symmetry (where $(\mathrm{S}(3)$ is the group of permutations of three objects) when $m_{1}(d)=m_{2}(d)=m_{1}(u)=m_{2}(u)=0 . \mathrm{M}_{c}$ has an $\mathrm{S}_{L}(2) \times \mathrm{S}_{R}(2)$ symmetry for the first two generations if $m_{1}(d)=m_{1}(u)=0$. As a consequence,

$$
\bar{q}_{c L} \mathrm{M}_{2 c} q_{c R}
$$

depends only on the quark fields

$$
q_{1 c}+q_{2 c} \text { and } q_{3 c}
$$

To get an idea of the pattern of breaking of the $\mathrm{S}_{L}(3) \times \mathrm{S}_{R}(3)$ symmetry due to $m_{2}(d) \neq 0$ and $m_{2}(u) \neq 0$ we look back at the case of two generations. There we noted that the symmetry breaking part changed sign when permuting the two quark fields in the coherent basis. We propose now in the present context a definite pattern of symmetry breaking in the form of Assumption 2.

Assumption 2:

The matrix $M_{2 c}$ changes sign under the transformation (both for $d$ and $u$ quarks)

$$
\frac{q_{1 c}+q_{2 c}}{\sqrt{2}} \leftrightarrow q_{3 c}
$$

It follows then from (16) that

$$
B=-2 c-\frac{b}{\sqrt{2}}=0
$$




$$
c=-\frac{b}{2 \sqrt{2}}
$$

With reference to equation $\left(6^{\prime}\right)$, we note that the sign of $b$ is not determined since we can change $q_{2} \rightarrow-q_{2}$ which changes $b \rightarrow-b$. Therefore the above condition is more generally

$$
b^{2}=8 c^{2}
$$

We remark that while we were led to Assumption 2 by the symmetry breaking for the first two generations (equation (11)), we treat the second and third generations by first transforming to real matrices $\mathrm{M}_{2}(d)$ and $\mathrm{M}_{2}(u)$. Different proposals for this symmetry breaking were made by Tanimoto [6] and by Fritzsch and Holtmannspötter [7].

5. We still keep $m_{1}(d)=m_{1}(u)=0$ and diagonalize $\mathrm{M}(d)$ and $\mathrm{M}(u)$ by different rotations in the $(2,3)$-plane to

$$
\mathrm{M}_{\text {diag }}=\operatorname{diag}\left(0,-m_{2}, m_{3}\right) .
$$

Using equation (18), the rotation angle $\theta_{23}^{(0)}$ is found to be

$$
\tan \theta_{23}^{(0)}=\frac{1}{4 \sqrt{2}}\left[\sqrt{\left(1+\frac{m_{2}}{m_{3}}\right)^{2}+32 \frac{m_{2}}{m_{3}}}-\left(1+\frac{m_{2}}{m_{3}}\right)\right]
$$

for $b>0$. We note that if we expand $\theta_{23}^{(0)}$ in powers of $\frac{m_{2}}{m_{3}}$

$$
\theta_{23}^{(0)}=O\left(\frac{m_{2}}{m_{3}}\right)
$$

Since $\left|V_{12}^{(2)}\right|=O\left(\sqrt{\frac{m_{1}}{m_{2}}}\right)$ by equation (9), and the mass ratios $\frac{m_{1}}{m_{2}}$ and $\frac{m_{2}}{m_{3}}$ are not very different, this makes it plausible that

$$
\left|V_{23}\right| \simeq\left|V_{12}\right|^{2}
$$

consistent with Wolfenstein's form [8]. To give an impression of the mixing between second and third generations which follows from our ansatz, we state that with

$$
\frac{m_{s}}{m_{b}}=0.025 \text { and } \quad \frac{m_{c}}{m_{t}}=0.005
$$

we obtain from (19)

$$
\left|V_{23}\left(m_{1}=0\right)\right|=0.045 \text {. }
$$

The correction due to $m_{1} \neq 0$ (which is given approximately by $\frac{m_{2}}{m_{3}} \rightarrow \frac{m_{2}}{m_{3}}\left(1-\frac{m_{1}}{m_{2}}\right)$, see below) lowers this value by about 5\%. To obtain agreement with the experimental information on $\left|V_{23}\right|$ we have to choose a value for $\frac{m_{s}}{m_{b}}$ near the lower end of the theoretical estimates [9] of this mass ratio. 
6. We now include the masses of the first generation. In this case

$$
\mathrm{M}=\mathrm{M}_{3}+\mathrm{M}_{2}+\mathrm{M}_{1}
$$

for both $M(d)$ and $M(u)$. In the heavy basis equation (6') still holds for $\mathrm{M}_{2}$. Equation (5) for $\mathrm{M}_{3}$ is slightly modified to

$$
\mathrm{M}_{3}=\left(m_{1}-m_{2}+m_{3}\right)\left(\begin{array}{ccc}
0 & 0 & 0 \\
0 & 0 & 0 \\
0 & 0 & 1
\end{array}\right)
$$

where the minus sign is due to our choice of a negative eigenvalue. In the spirit of Assumption 2, we propose the following pattern for the symmetry breaking caused by $m_{1}(d) \neq 0$, $m_{1}(u) \neq 0$.

Assumption 3:

$\mathrm{M}_{1 c}$ transforms as the antisymmetric representation of a subgroup $\overline{\mathrm{S}}(3)$ (isomorphic to the permutation group $\mathrm{S}(3))$ of $\mathrm{S}_{L}(3) \times \mathrm{S}_{R}(3)$. $\overline{\mathrm{S}}(3)$ must include among its elements

$$
\begin{aligned}
\overline{1} & =1_{L} \cdot 1_{R} \\
\overline{(12)} & =(12)_{L} \cdot(12)_{R}
\end{aligned}
$$

where (12) denotes the transposition of 1 and 2.

This Assumption 3 is motivated by the considerations of section 3, namely the antisymmetry under the transformation $q_{1 c} \leftrightarrow q_{2 c}$ expressed by equation (11). From Assumption 3 we find that there are only two possibilities for $\mathrm{M}_{1 c}$ :

(i) $\overline{\mathrm{S}}(3)$ is the diagonal subgroup. Then it follows that

$$
\mathrm{M}_{1 c}=\text { constant } \cdot\left(\begin{array}{ccc}
0 & i & -i \\
-i & 0 & i \\
i & -i & 0
\end{array}\right)
$$

(ii) $\overline{\mathrm{S}}(3)$ has the elements

$$
\left(p_{i}^{-1}\right)_{L} \cdot\left((12) p_{i}(12)\right)_{R},
$$

where $p_{i}(i=1,2, \cdots, 6)$ is an element of $\mathrm{S}(3)$. Then

$$
\mathrm{M}_{1 c}=\text { constant } \cdot\left(\begin{array}{ccc}
1 & 0 & -1 \\
0 & -1 & 1 \\
-1 & 1 & 0
\end{array}\right) \text {. }
$$

In the heavy basis these two $\mathrm{M}_{1}$ 's are respectively

$$
\mathrm{M}_{1}=d \cdot\left(\begin{array}{ccc}
0 & i & 0 \\
-i & 0 & 0 \\
0 & 0 & 0
\end{array}\right)
$$


and

$$
\mathrm{M}_{1}=d \cdot\left(\begin{array}{lll}
0 & 1 & 0 \\
1 & 0 & 0 \\
0 & 0 & 0
\end{array}\right)
$$

This agrees with the result for the first two generations in the limit $m_{3} \rightarrow \infty$ stated in equations (7) and (9) if we choose (i) for the $u$-quarks and (ii) for the $d$-quarks or vice versa. It supports the idea that $\phi=\frac{\pi}{2}$.

7. To summarize, our scheme of symmetry breaking has led to the following mass matrices:

$$
\mathrm{M}(d)=\left(\begin{array}{ccc}
0 & d(d) & 0 \\
d(d) & c(d) & b(d) \\
0 & b(d) & a(d)
\end{array}\right)
$$

and

$$
\mathrm{M}(u)=\left(\begin{array}{ccc}
0 & i d(u) & 0 \\
-i d(u) & c(u) & b(u) \\
0 & b(u) & a(u)
\end{array}\right)
$$

where

$$
\begin{aligned}
& b^{2}(d)=8 c^{2}(d) \\
& b^{2}(u)=8 c^{2}(u) .
\end{aligned}
$$

We can change $d(d) \rightarrow i d(d), i d(u) \rightarrow d(u)$. With (25) and (26), we have expressed $\mathrm{M}(d)$ and $\mathrm{M}(u)$ in terms of three real parameters each; they can be expressed in terms of the six quark masses. The presence of $i$ in one of the matrices (25) or (26), which implies $C P$-nonconservation, is needed for agreement with the experimental value of the Cabibbo angle. This connection has been noted before [10].

8. The diagonalization of mass matrices (25) and (26) to $\mathrm{M}_{\text {diag }}=\operatorname{diag}\left(m_{1},-m_{2}, m_{3}\right)$ is performed by

$$
\begin{aligned}
& \mathrm{M}(d)=R(d) \mathrm{M}_{\text {diag }}(d) R^{T}(d) \\
& \mathrm{M}(u)=\operatorname{diag}(-i, 1,1) R(u) \mathrm{M}_{\text {diag }}(u) R^{T}(u) \operatorname{diag}(i, 1,1)
\end{aligned}
$$

where $R(d)$ and $R(u)$ are orthogonal matrices. The expressions for $a, c, d$ in terms of the eigenvalues are

$$
\begin{aligned}
a+c & =m_{3}-m_{2}+m_{1} \\
8 c^{2}+d^{2}-a c & =m_{3} m_{2}-m_{3} m_{1}+m_{2} m_{1} \\
a d^{2} & =m_{1} m_{2} m_{3}
\end{aligned}
$$

both for $d$ and $u$ quarks. This leads to a cubic equation for the parameters.

We simplify these equations by an approximation which gives the elements of $V$ to sufficient accuracy. We neglect the small term $\frac{\left(a-m_{3}\right) d^{2}}{m_{3}^{3}}$. Then equation $(28)$ is approximated by

$$
\begin{aligned}
a+c & =m_{3}-m_{2}+m_{1} \\
8 c^{2}-a c & =m_{3} m_{2}-m_{3} m_{1} \\
d^{2} & =m_{1} m_{2} .
\end{aligned}
$$


Equation (29) leads to a simple form of the rotation matrices (27),

$$
R=R_{23}\left(\theta_{23}\right) \cdot R_{12}\left(\theta_{12}\right)
$$

where $R_{12}$ and $R_{23}$ are rotations in the 1-2 and 2-3 planes respectively. A mass matrix which can be diagonalized by a rotation (30) was proposed by Tanimoto [6], who chose $\mathrm{M}_{1}$ such that $(30)$ is an exact relation. His $R_{23}$ is different from ours. Explicitly,

$$
R_{12}=\left(\begin{array}{ccc}
\cos \theta_{12} & +\sin \theta_{12} & 0 \\
-\sin \theta_{12} & \cos \theta_{12} & 0 \\
0 & 0 & 1
\end{array}\right)
$$

and

$$
R_{23}=\left(\begin{array}{ccc}
1 & 0 & 0 \\
0 & \cos \theta_{23} & +\sin \theta_{23} \\
0 & -\sin \theta_{23} & \cos \theta_{23}
\end{array}\right)
$$

with

$$
\tan \theta_{12}=\sqrt{\frac{m_{1}}{m_{2}}}
$$

and

$$
\tan \theta_{23}=\frac{1}{4 \sqrt{2}}\left[\sqrt{\left(1+\frac{m_{2}-m_{1}}{m_{3}}\right)^{2}+32\left(\frac{m_{2}-m_{1}}{m_{3}}\right)}-\left(1+\frac{m_{2}-m_{1}}{m_{3}}\right)\right] .
$$

This leads to an approximate unitary KM matrix

$$
\begin{aligned}
\tilde{V} & =R_{12}^{T}(u) R_{23}^{T}(u) \operatorname{diag}(i, 1,1) R_{23}(d) R_{12}(d) \\
& =\left(\begin{array}{ccc}
i c_{1} c_{2}+s_{1} s_{2} c_{3} & i c_{1} s_{2}-s_{1} c_{2} c_{3} & -s_{1} s_{3} \\
i s_{1} c_{2}-c_{1} s_{2} c_{3} & i s_{1} s_{2}+c_{1} c_{2} c_{3} & c_{1} s_{3} \\
s_{2} s_{3} & -c_{2} s_{3} & c_{3}
\end{array}\right)
\end{aligned}
$$

where

$$
\begin{array}{lll}
c_{1}=\cos \theta_{12}(u) & c_{2}=\cos \theta_{12}(d) & c_{3}=\cos \left(\theta_{23}(d)-\theta_{23}(u)\right) \\
s_{1}=\sin \theta_{12}(u) & s_{2}=\sin \theta_{12}(d) & s_{3}=\sin \left(\theta_{23}(d)-\theta_{23}(u)\right) .
\end{array}
$$

9. Remarks

a) The $\tilde{V}$ given by equation (35) has only three parameters. Therefore, the absolute values of its matrix elements satisfy one relation:

$$
\begin{aligned}
& \left|\tilde{V}_{12}\right|^{2}\left[\left|\tilde{V}_{31}\right|^{2}+\left|\tilde{V}_{32}\right|^{2}\right]^{2}+\left|\tilde{V}_{13}\right|^{2}\left[\left|\tilde{V}_{31}\right|^{2}-\left|\tilde{V}_{32}\right|^{2}\right] \\
- & {\left[\left|\tilde{V}_{31}\right|^{2}-\left|\tilde{V}_{13}\right|^{2}\left|\tilde{V}_{32}\right|^{2}\right]\left[\left|\tilde{V}_{31}\right|^{2}+\left|\tilde{V}_{32}\right|^{2}\right]=0 . }
\end{aligned}
$$

b) It follows from equation (35) that [11]

$$
\tilde{J}_{C P}=\operatorname{Im}\left(\tilde{V}_{11} \tilde{V}_{33} \tilde{V}_{13}^{*} \tilde{V}_{31}^{*}\right)=-s_{1} c_{1} s_{2} c_{2} s_{3}^{2} c_{3} .
$$


The sign of $\tilde{J}_{C P}$ is undetermined since we can change $i \rightarrow-i$ in equation (35).

10. We conclude with some sample numerical values. For

$$
\begin{array}{ll}
\frac{m_{d}}{m_{s}}=0.05 & \frac{m_{s}}{m_{b}}=0.025 \\
\frac{m_{u}}{m_{c}}=0.004 & \frac{m_{c}}{m_{t}}=0.005
\end{array}
$$

we get

$$
\begin{gathered}
\left|V_{12}\right|=0.226 \\
\left|V_{23}\right|=0.043 \\
\frac{\left|V_{13}\right|}{\left|V_{23}\right|}=0.06 \\
\left|J_{C P}\right|=2.6 \times 10^{-5} .
\end{gathered}
$$

\section{Acknowledgements}

CN and TTW acknowledge the kind hospitality of the Theory Division at CERN. TTW thanks Maurice Jacob for useful discussions. 


\section{References}

[1] M. Kobayashi and T. Maskawa, Prog. Theor. Phys. 49, 652 (1973).

[2] H. Fritzsch, Phys. Lett. B184, 391 (1987).

[3] H. Harari, H. Hant and J. Weyers, Phys. Lett. B78, 459 (1978);

P. Kaus and S. Meshkov, Mod. Phys. Lett. A3, 1251 (1988),

and Phys. Rev. D42, 1863 (1990);

H. Fritzsch and J. Plankl, Phys. Lett. B237, 451 (1990).

[4] H. Fritzsch, Nucl. Phys. B155, 189 (1979).

[5] N. Cabibbo, Phys. Rev. Lett. 10, 531 (1963).

[6] M. Tanimoto, Phys. Rev D41, 1586 (1990).

[7] H. Fritzsch and D. Holtmannspötter, Phys. Lett. B338, 290 (1994).

[8] L. Wolfenstein, Phys. Rev. Lett. 51, 1945 (1983).

[9] J. Gasser and H. Leutwyler, Phys. Rep. 87, 77 (1982);

Y. Koide, preprint US-94-05 (1994).

[10] M. Shin, Phys. Lett. B145, 285 (1984);

M. Gronau, R. Johnson and J. Schechter, Phys. Rev. Lett. 54, 2176 (1985);

H. Fritzsch and Z. Xing, Phys. Lett. B353, 114 (1995).

[11] C. Jarlskog, Phys. Rev. Lett. 55, 1039 (1985),

and Zeit. f. Physik C-Particles and Fields 29, 491 (1985). 